\title{
Multi-wavelength extragalactic astronomy at Ghent University
}

\author{
Maarten Baes, Peter Camps, Ilse De Looze, Sébastien Viaene
}

Sterrenkundig Observatorium, Universiteit Gent, Krijgslaan 281 S9, 9000 Gent, Belgium

\begin{abstract}
Galaxies are generally considered as the basic building blocks of the Universe. Besides stars, galaxies also contain a supermassive black hole, a dark matter halo, and a multi-phase interstellar medium that consists of molecules, atoms, ions, and dust grains. A global picture of the structure, dynamics, formation, and evolution of galaxies requires observations of all these different components, and hence a multi-wavelength approach. We describe the main contributions of the UGent astronomy group to extragalactic astronomy over the last years. We focus on two broad and slightly overlapping fields in which our team has been active: galaxy kinematics and dynamics, and the study of the interstellar medium in galaxies. Possibilities for collaboration with the Indian astronomical community in the frame of the BINA network are highlighted.
\end{abstract}

Keywords: galaxies; structure and dynamics - galaxies: evolution - galaxies: ISM

\section{Introduction}

With more than 44,000 students and 15,000 staff members, Ghent University (UGent) is one of the largest universities in the Low Countries, and an active partner in national and international educational, scientific and industrial cooperation. Both in terms of education and research, UGent is consistently ranked among the best universities in Belgium and worldwide. 11

Astronomy and astrophysics research at UGent is done at the Sterrenkundig Observatorium, a research group within the Department of Physics and Astronomy. We are primarily involved in extragalactic astronomy and cosmology. More specifically, we study the structure, dynamics, and evolution of galaxies, using both observational and theoretical methodologies. In this contribution, we present an inexhaustive overview of the activities and major contributions of our UGent astronomy group to two broad subdomains in extragalactic astronomy: galaxy kinematics and dynamics (Section 2), and the study of the dusty interstellar medium in galaxies (Section 3). In Section 4 we discuss possible avenues of cooperation with the Indian astronomical community in the frame of the BINA network.

\section{Galaxy kinematics and dynamics}

Galactic dynamics is the study of the motions of the stars and gas in order to explain the main morphological and kinematical features of galaxies. Stellar orbits are governed purely by their gravitational forces, and by studying line-of-sight stellar kinematics, one can hope to determine the mass

${ }^{1}$ For example, http://www. shanghairanking.com 
distribution in galaxies. The study of stellar orbits to form self-consistent statistical mechanical configurations, is called stellar dynamics, and this constitutes the central approach to galactic dynamics. Gas dynamics, on the other hand, is governed also by dissipative forces, which can, to some extent, influence particular morphological and kinematical features of galaxies.

\subsection{Stellar dynamics}

Stellar dynamics was the main research topic at UGent when astronomy research was first developed in the 1980s. Our group has been particularly active in the construction of self-consistent equilibrium models for galaxies. Over the years, new inversion techniques and advanced analytical tools have been explored to construct self-consistent analytical toy models for galaxies, and investigate their photometric, kinematical and dynamical properties (Dejonghe 1987; Baes \& Dejonghe 2002b; Baes \& Van Hese 2007, 2011; Van Hese et al. 2009). Such models are useful as simple fitting tools on their own, or as starting point for theoretical studies or numerical simulations. Typical examples are the study of the effect of dust attenuation on the observed kinematics of galaxies (Baes \& Dejonghe 2000, 2001c, 2002a; Baes et al. 2000), and linear mode analysis to study instabilities in disc galaxies (De Rijcke \& Voulis 2016; De Rijcke et al. 2019).

In the late 1980s, a numerical code to fit dynamical equilibrium models to observational photometric and kinematical data sets was developed (Dejonghe 1989). The models were constructed as a linear superposition of fully analytical components, with the coefficients of the linear combination determined by a quadratic programming technique. This code was widely applied to study the kinematical and dynamical structure of different types of galaxies (Dejonghe et al. 1996; De Bruyne et al. 2001, 2004), and to search for and characterise the properties of dark matter haloes around early-type galaxies (Saglia et al. 1993; Mathieu et al. 1996; Statler et al. 1999; Emsellem et al. 1999). Unfortunately, the software tool was not further developed and, since the mid-2000s, other approaches such as the Schwarzschild orbit superposition method or the particle-based made-to-measure method have become more popular in the field (e.g., Cappellari et al. 2006; Long \& Mao 2012; Zhu et al. 2014).

\subsection{Galaxy kinematics at intermediate redshifts}

With the increasing power of telescopes, it has become possible to perform stellar dynamics studies at higher redshifts. Astronomers from our group are leading LEGA-C. $2^{2}$ an ESO Public Spectroscopic Survey that has obtained deep continuum spectroscopy of several thousand galaxies at redshifts 0.6 to 1 (van der Wel et al. 2016; Straatman et al. 2018); see Figure1. The observations started in 2014 and were completed in 2018, covering 128 nights in total. These deep, high resolution spectra, combined with deep imaging, provide very detailed data of stellar continua for galaxies at half the Universe's age: stellar ages, masses, metallicities, stellar kinematics, gas kinematics, and star formation histories (Wu et al. 2018a,b; Chauke et al. 2018; Spilker et al. 2018; Bezanson et al. 2018a,b).

LEGA-C enables for the first time several studies of key questions in galaxy evolution on long cosmological time scales (up to $8 \mathrm{Gyr}$ ), especially for early-type galaxies: dynamical evolution, mass assembly through merging and star formation, and the mechanisms that quench star formation. Bezanson et al. (2018a) recently showed that the most massive LEGA-C galaxies generally show no or little rotation, whereas less massive galaxies show significant rotation. Comparing the LEGA-C galaxies to local galaxies, it was found that quiescent galaxies must lose angular momentum between $z \sim 1$ and the present, presumably through dissipationless merging, and/or that the mechanism that transforms star-forming galaxies also reduces their rotational support.

\footnotetext{
${ }^{2}$ http://www.mpia.de/home/legac/
} 

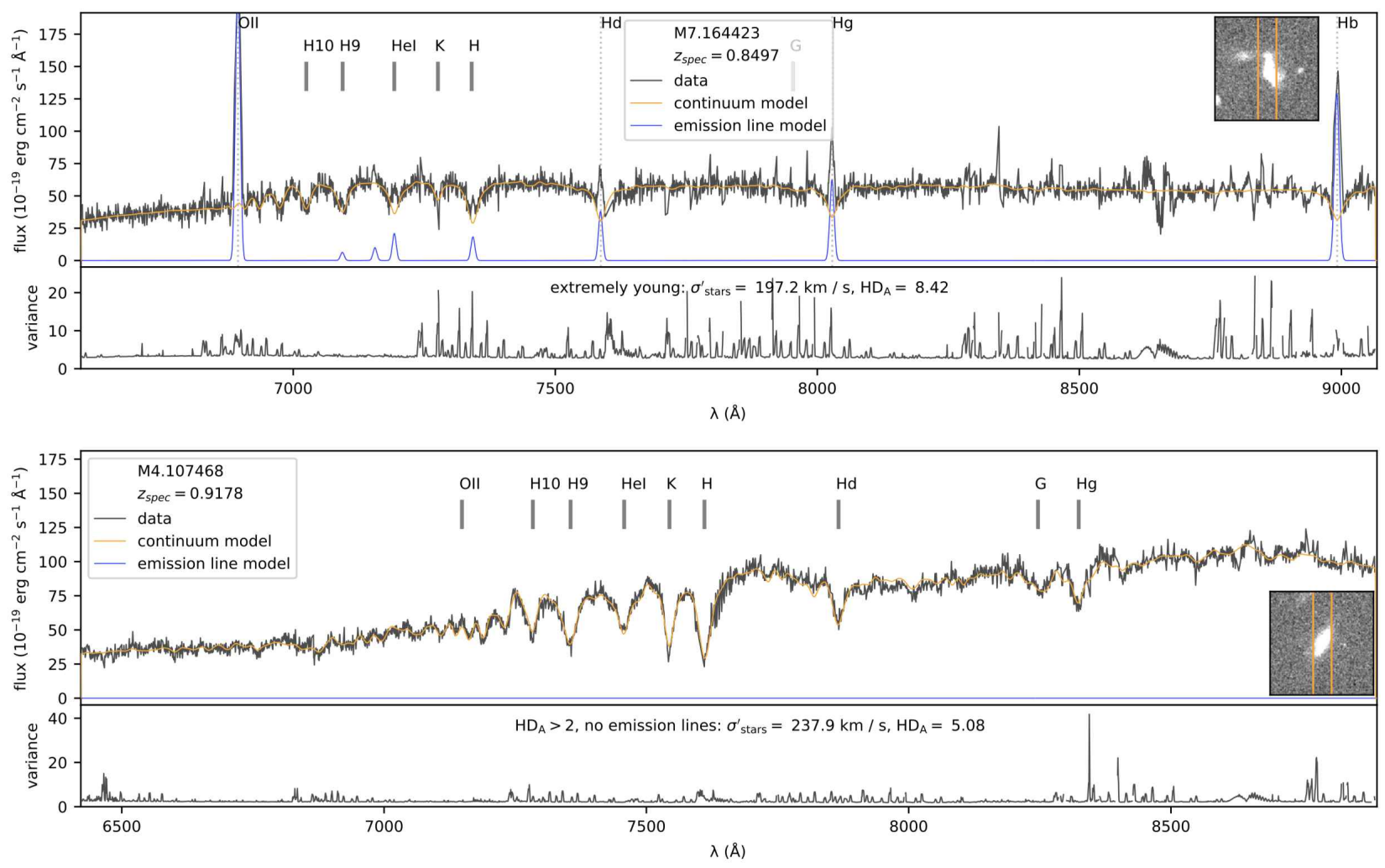

Figure 1: Two examples of LEGA-C galaxies, showing the high-quality optical spectra with the bestfit continuum and emission-line models. The top panel corresponds to an extremely young galaxy with strong emission lines, the bottom panel to a more massive galaxy with no emission line contribution. Figure adapted from Straatman et al. (2018).

\subsection{Gas kinematics and gravitational lensing}

Apart from stellar dynamics, there are other means to determine the mass distribution, and particularly the presence of dark matter, within and around galaxies. For spiral galaxies, the kinematics of interstellar gas have been used since the late 1970s to search for dark matter, and both ionised gas and neutral hydrogen gas have been adopted as a useful tracer. We have mainly focused on HI $21 \mathrm{~cm}$ observations to trace the gas content and the outer kinematics of disc galaxies. We have been involved in several large Hi projects such as the AGES project (Auld et al. 2006; Cortese et al. 2008) and the HALOGAS survey (Heald et al. 2011; Gentile et al. 2013; de Blok et al. 2014), and many smallerscale projects targeting smaller samples or individual galaxies (Gentile et al. 2010, 2015; Allaert et al. 2015). A recent study by Allaert et al. (2017) showed that observed Hi rotation curves are in agreement with theoretical predictions of cosmological simulations, which suggest that baryonic processes, and in particular supernova feedback following bursts of star formation, can alter the structure of dark matter haloes and transform primordial cusps into shallower cores.

Finally, gravitational lensing is an additional useful method to investigate the mass distribution in galaxies. Our team has contributed to this field by analytically investigating the lensing properties of important toy models (Retana-Montenegro et al. 2012a,b), and by constructing a non-parametric inversion method of strong gravitational lensing systems based on genetic algorithms (Liesenborgs et al. 2006, 2007; Liesenborgs \& De Rijcke 2012). Gravitational lensing is particularly valuable at submillimeter wavelengths for the study of the statistical and individual properties of dusty starforming galaxies, and a large number of strongly lensed star-forming galaxies has been detected in 
a

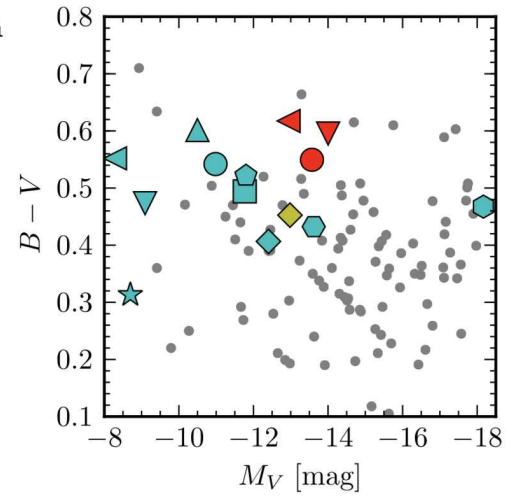

d

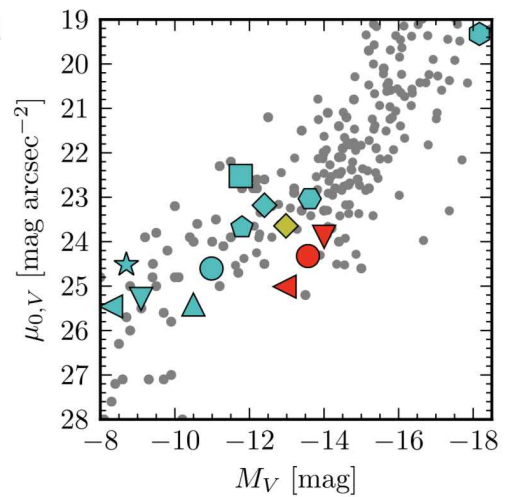

b

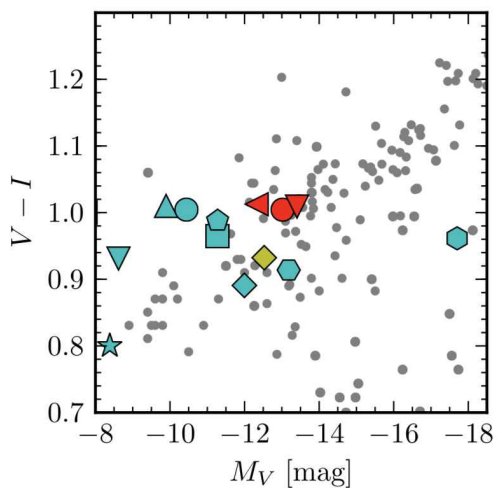

e

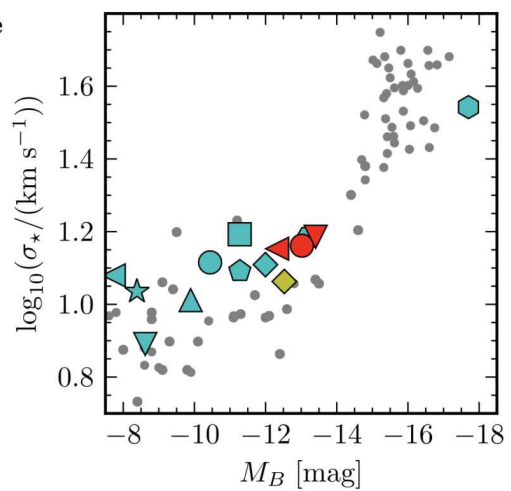

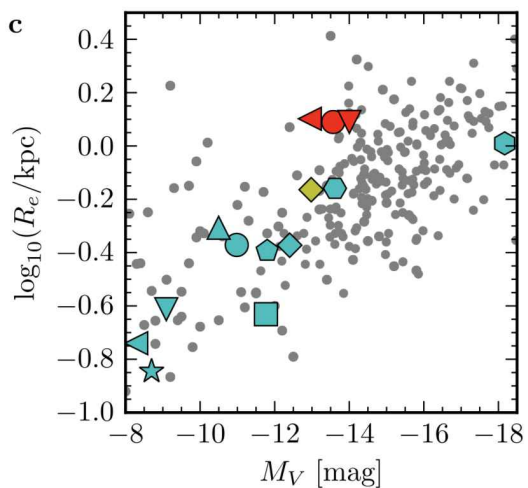

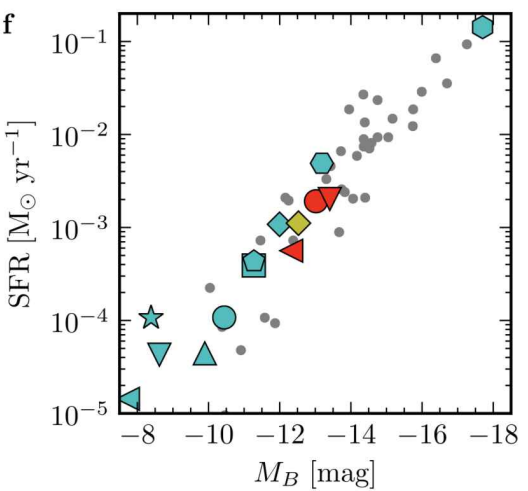

Figure 2: Scaling relations for dwarf galaxies. The grey dots correspond to observations, the coloured symbols correspond to the galaxies simulated by Verbeke et al. (2015).

wide-area submillimeter surveys (Negrello et al. 2010, 2017). The detailed analysis of these sources, through follow-up observations and detailed lens modelling, proves to be an important way to probe star formation in the high-redshift Universe (Bussmann et al. 2012; Calanog et al. 2014; Timmons et al. 2016; Amvrosiadis et al. 2018; Dye et al. 2018).

\subsection{Numerical simulations of galaxies}

A final research topic at UGent that is connected to galaxy dynamics is the use of high-performance numerical simulations to investigate the evolution of the mass distribution, morphology and kinematics of galaxies. One research line is the application of N-body/hydrodynamics simulations to study the evolution of dwarf galaxies. Dwarf galaxy research kicked off with an ESO Large Program on the internal dynamics of dwarf elliptical galaxies in the Fornax Cluster and in nearby galaxy groups (De Rijcke et al. 2001, 2005; Koleva et al. 2009). This program led to the need to perform numerical simulations in order to try and understand the observations. Over the past few years, the simulations, based on an extended version of the GADGET-2 SPH code, have become increasingly sophisticated, and include effects as composition-dependent cooling and heating curves, dwarf galaxy merger trees and feedback from population III stars (Valcke et al. 2008; Schroyen et al. 2011, 2013; Verbeke et al. 2015). The simulations generally reproduce the observed scaling laws very well (Figure 2). Using the latest suite of MORIA simulations, Verbeke et al. (2017) demonstrated that low-mass late-type dwarf galaxies inhabit more massive dark matter halos than would be inferred from their kinematics, an important finding in the context of the notorious Too Big To Fail problem.

On a physically larger scale, we are involved in EAGLE, a suite of hydrodynamical simulations that follow the formation of galaxies and supermassive black holes in cosmologically representa- 


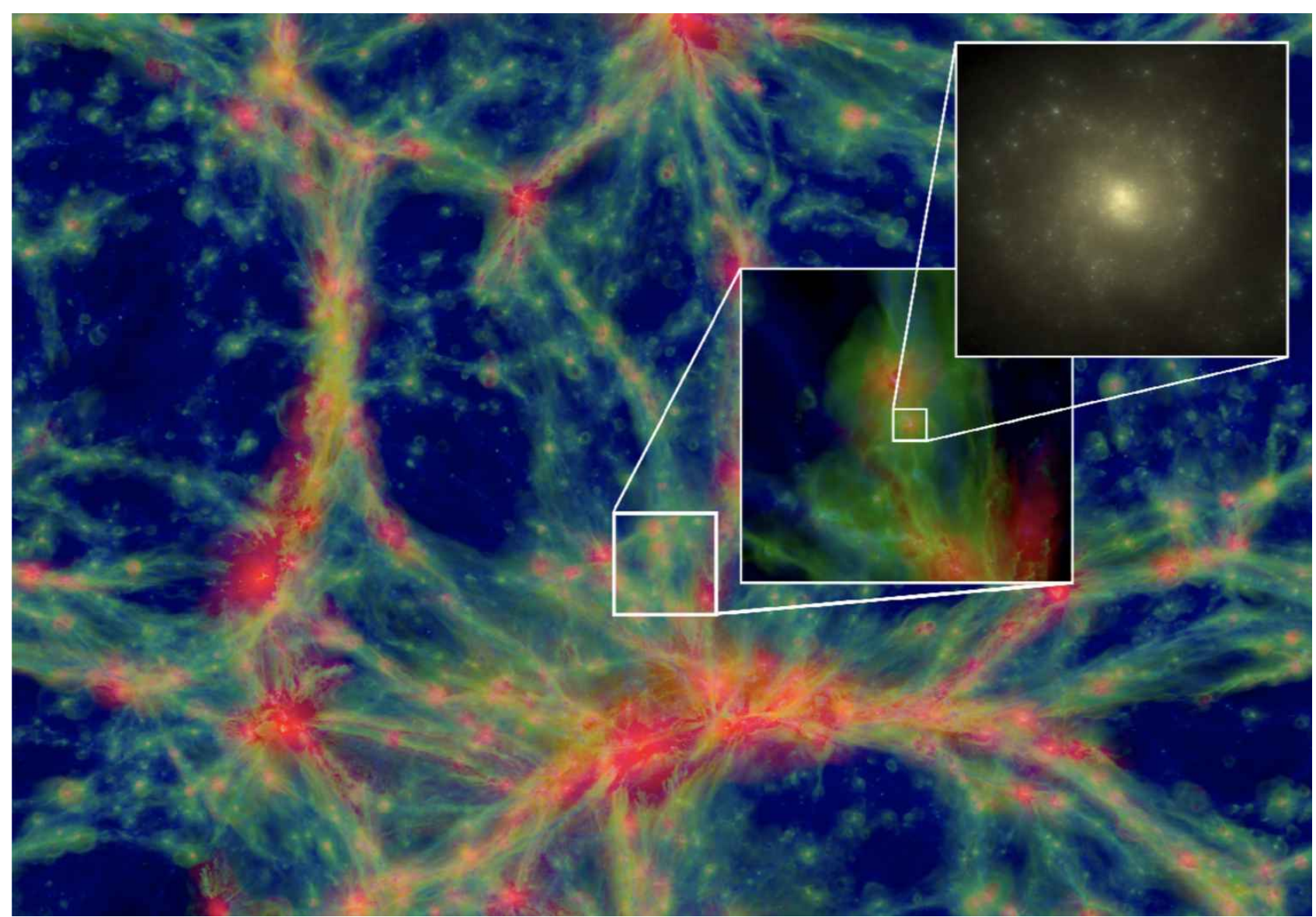

Figure 3: A slice through the reference EAGLE cosmological simulation at redshift $z=0$. The intensity shows the gas density while the colour encodes the gas temperature. The insets show regions of $10 \mathrm{Mpc}$ and $60 \mathrm{kpc}$ on a side and zoom into an individual galaxy. The deepest zoom image shows the simulated ugr three-color image created using the SKIRT radiative transfer code. Figure adapted from Schaye et al. (2015).

tive volumes of a standard $\Lambda$ CDM universe (Schaye et al. 2015). The EAGLE simulations have been calibrated to reproduce the local Universe stellar mass function, the galaxy-central black hole mass relation, and the galaxy mass-size relation. The simulations were shown to be in reasonable to excellent agreement with many other observables not considered in the calibration. Our group's contributions to this project are predominantly linked to the comparison of the EAGLE simulation to the observed universe through synthetic multi-wavelength data (Camps et al. 2016, 2018; Trayford et al. 2017; Baes et al. 2019), see Figure 3 ,

\section{Interstellar dust in galaxies}

The second broad research theme at UGent is the study of the dusty interstellar medium (ISM) in galaxies. Although dust only makes up a small part of the interstellar material (typically $1 \%$ in mass), its impact on the other baryonic components in galaxies should not be underestimated. For example, dust particles act as a catalyst for the formation of molecular hydrogen, regulate the heating of the neutral gas component through photoelectric heating and inelastic interactions, and provide shielding for molecules from the UV radiation of young stars. On galaxy-wide scales, interstellar dust affects our view on galaxies: on average, about 30 to $50 \%$ of the starlight in the Universe is absorbed by 
interstellar dust grains, and converted to FIR/submm emission (Davies et al. 2012; Viaene et al. 2016; Bianchi et al. 2019). It is hence important to carefully trace and map the dust in galaxies of different types and in different environments.

\subsection{Far-infrared observations of galaxies}

The most direct way to trace and characterise the interstellar dust component in galaxies is through its thermal emission in the far-infrared. The Herschel Space Observatory, operational from 2009 to 2013, opened a new window on the FIR/submm spectral domain, allowing astronomers to probe the cold dust component in a large number of nearby and distant objects. Many Herschel programmes were devoted to galaxies, and our group at UGent has been deeply involved with several of these. Some were targeted surveys that focused on different samples of nearby galaxies (Boselli et al. 2010; Verstappen et al. 2013; Madden et al. 2013). Other projects were blind surveys that mapped large areas of extragalactic sky, which can be used to investigate the dust properties of nearby and distant galaxies in a more statistical way (Davies et al. 2010; Eales et al. 2010; Dunne et al. 2011; De Vis et al. 2017).

One of the major achievements of Herschel on nearby galaxies is a solid characterisation of the cool dust budget of galaxies of different types and in different environments. The Herschel Reference Survey (Boselli et al. 2010) has imaged more than 300 stellar-mass-selected galaxies and allowed us to investigate the dust scaling relations along the Hubble sequence (Cortese et al. 2012; Boselli et al. 2012; Smith et al. 2012a). This project expanded into the DustPedia project. ${ }^{3}$ an EU FP7 project in which UGent was one of the leading institutes (Davies et al. 2017; Clark et al. 2018). The goal of DustPedia was to combine Herschel and Planck with ancillary data to make an extensive and intensive study of the stars, gas and dust in almost 1000 galaxies in the nearby Universe (Casasola et al. 2017; Bianchi et al. 2018; De Vis et al. 2019; Nersesian et al. 2019).

The superior spatial resolution of Herschel also enables studies of the distribution and heating sources of dust within nearby galaxies, and many prototypical galaxies have been or are being examined in detail (Bendo et al. 2010, 2012; Baes et al. 2010b; De Looze et al. 2012c; Hughes et al. 2014, 2015, 2016). One of the most spectacular and iconic images of the Herschel mission is the $5.5 \times 2.5$ $\mathrm{deg}^{2} \mathrm{FIR} / \mathrm{submm}$ image of the Andromeda galaxy, taken in the frame of the HELGA programme (Fritz et al. 2012). These images, combined with ancillary multi-wavelength imaginery from UV to radio wavelengths (Figure 4), enable a detailed investigation of the dust heating mechanisms and dust scaling relations on local scales of only 140 pc (Smith et al. 2012; Ford et al. 2013; Viaene et al. 2014, 2017a,b).

Besides Herschel, we also make use of other far-infrared and submm facilities to observe the dusty ISM in galaxies, including Planck (Herranz et al. 2013; Baes et al. 2014; Greenslade et al. 2018), ALMA (Indebetouw et al. 2014; Hughes et al. 2017a,b; Dye et al. 2018) and the JCMT (Bakx et al. 2018; Saintonge et al. 2018). We also look forward to the next-generation ESA/JAXA far-infrared mission SPICA, which is proposed for launch around 2032 and would enable to study the dusty ISM in galaxies out to large redshifts (Spinoglio et al. 2017; Gruppioni et al. 2017; Fernández-Ontiveros et al. 2017; van der Tak et al. 2018).

\subsection{Attenuation and radiative transfer}

Apart from the thermal emission at FIR/submm wavelengths, a complementary way to investigate the amount and spatial distribution of interstellar dust in galaxies is by analysing its effect on the starlight at UV and optical wavelengths. The proper way to do this is by means of radiative transfer

$\sqrt[3]{\text { http: / / www.dustpedia.com }}$ 


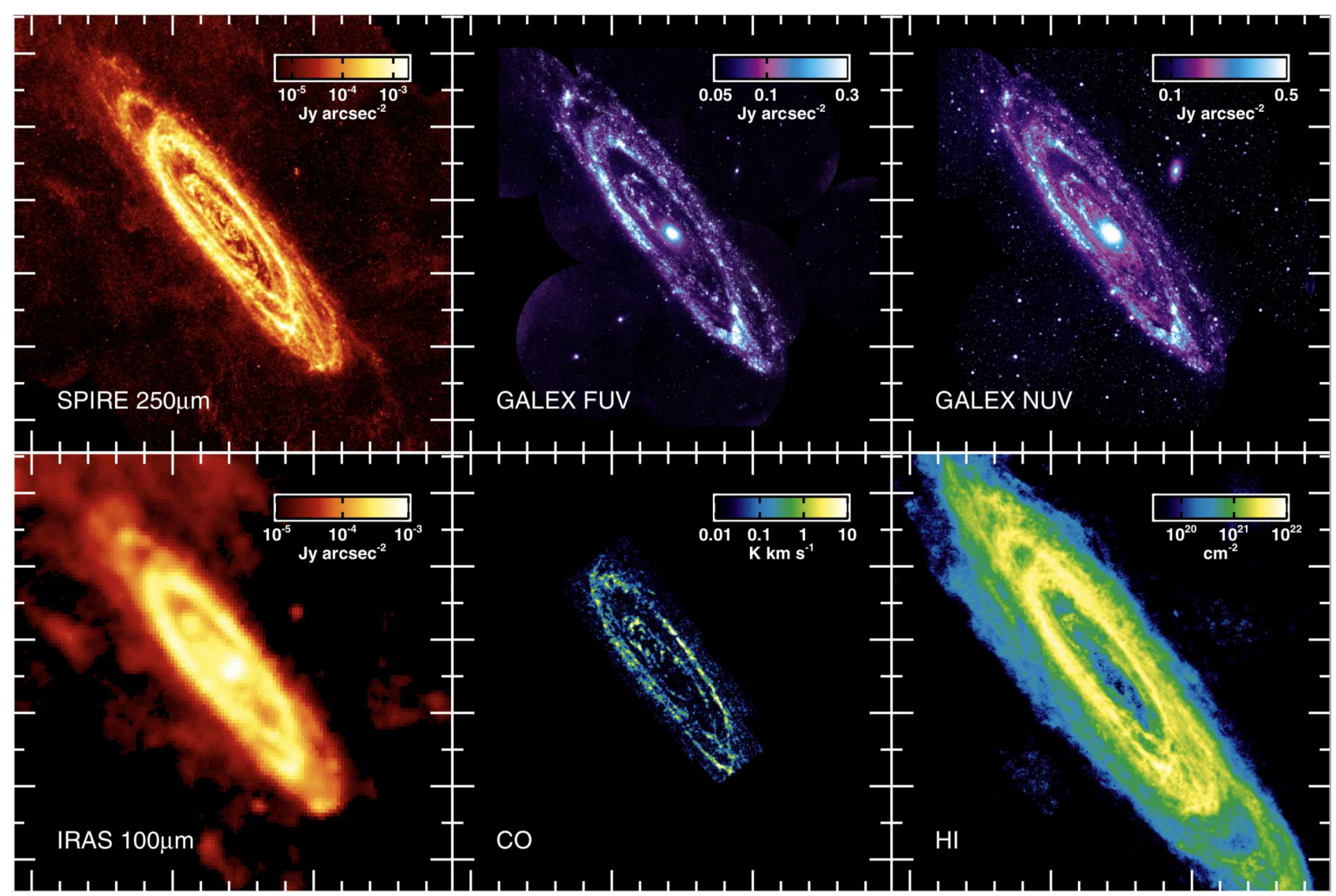

Figure 4: A multi-wavelength image collection of the Andromeda galaxy, put together in the frame of the HELGA project. Figure from Fritz et al. (2012).

calculations that take into account the relevant effects (absorption and multiple anisotropic scattering) in a realistic geometrical setting, i.e. through 3D radiative transfer. 3D radiative transfer is generally considered to be one of the remaining grand challenges in computational astrophysics (Steinacker et al. 2013).

Over the past two decades, we have investigated and compared different methods to deal with radiative transfer problems (Baes \& Dejonghe 2001a,b; Lee et al. 2016; Camps et al. 2018). We have developed and continuously improved a 3D Monte Carlo radiative transfer code, SKIRT ${ }^{4}$ (Baes et al. 2003, 2011; Camps \& Baes 2015). This code is nowadays a multi-purpose open-source code, equipped with advanced grids for spatial discretisation (Saftly et al. 2013, 2014; Camps et al. 2013), a hybrid parallelisation scheme (Verstocken et al. 2017), a library of input models (Baes \& Camps 2015), and a suite of optimisation techniques (Baes et al. 2003, 2005, 2011, 2016). It has been thoroughly benchmarked against other radiative transfer codes (Camps et al. 2015; Gordon et al. 2017). SKIRT is mainly used to model dusty galaxies, but has also been used for other applications, including dusty AGN tori (Stalevski et al. 2012, 2016, 2019), molecular clouds (Hendrix et al. 2015), and binary stellar systems (Deschamps et al. 2015; Hendrix et al. 2016).

\subsection{Radiative transfer modelling of nearby galaxies}

The availability of radiative transfer codes that can self-consistently model the attenuation and thermal emission by dust in 3D geometries opens up the possibility of dust energy balance studies of nearby

${ }^{4}$ http://www.skirt.ugent.be 


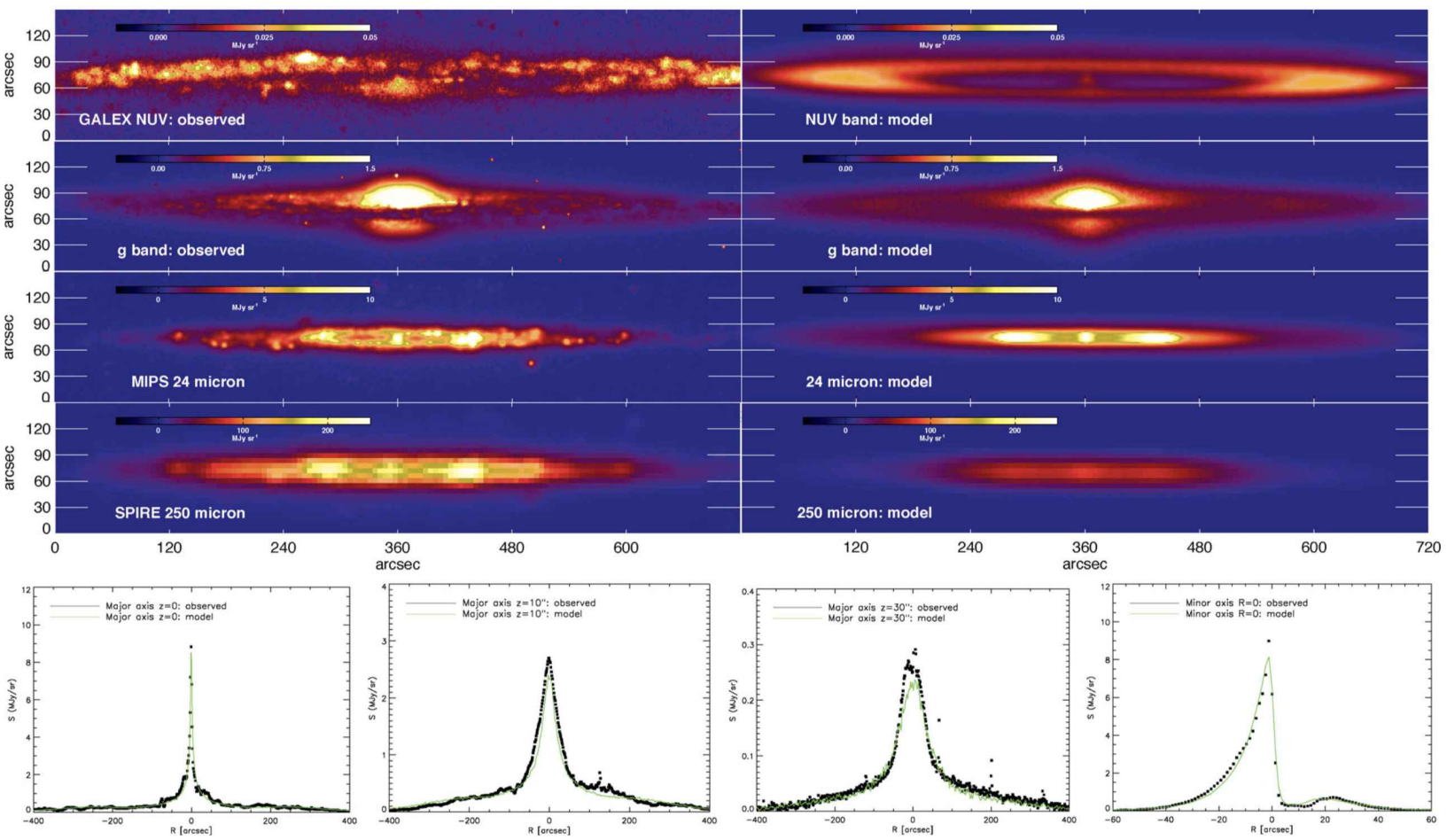

Figure 5: Radiative transfer modelling of the edge-on spiral galaxy NGC 4565 by De Looze et al. (2012a). The panels on the left are observed images from NUV to submm wavelengths, the panels on the right correspond to the model fit. The bottom panels show major and minor axis $g$-band profiles, showing the quality of the fit at optical wavelengths.

galaxies. As the starlight that is absorbed by dust grains in the UV-optical region is re-emitted in the infrared, one would expect the absorbed luminosity to be equal to the total thermal luminosity. Panchromatic radiative transfer modelling has mainly been applied to a number of edge-on spiral galaxies (Baes et al. 2010a; De Looze et al. 2012a,b; De Geyter et al. 2015; Baes \& Viaene 2016). These studies revealed a dust energy balance problem, in the sense that the predicted FIR fluxes of self-consistent radiative transfer models that successfully explain the optical extinction generally underestimate the observed FIR fluxes by a factor of about three (Figure 5). The most probable explanation is the large- and small-scale structure in the ISM (Saftly et al. 2015; Mosenkov et al. 2016, 2018).

More recently, we have also extended this panchromatic modelling effort to more face-on galaxies, which enables us to study the different dust heating agents in nearby galaxies (De Looze et al. 2014; Viaene et al. 2017a,b; Verstocken et al. 2019).

\section{Possibilities for BINA collaboration}

BINA, the Belgo-Indian Network for Astronomy and astrophysics (Joshi \& De Cat 2019), has the goal to foster collaboration between Belgian and Indian astronomy groups. At the moment, none of the Indian partners in the BINA network actively work on nearby galaxies, so collaboration between UGent and Indian BINA institutes does not look obvious.

The BINA collaboration is mainly centred around the new Indo-Belgian telescopes installed at the Devasthal observatory, in particular the optical-NIR 3.6m DOT telescope (Kumar et al. 2018). At this moment, this telescope with its current instrumentation suite does not really match our needs 
in the field of stellar kinematics, as stellar kinematics studies of dwarf galaxies or galaxies at intermediate redshift typically require $8 \mathrm{~m}$ class telescopes (De Rijcke et al. 2001; van der Wel et al. 2016). However, it can be used for deep broadband or narrowband imaging of nearby galaxies for multi-wavelength radiative transfer modelling (Mosenkov et al. 2016, 2018). The integral field spectrograph DOTIFS (Chung et al. 2018), currently under construction, would open up interesting possibilities for stellar kinematics, stellar population synthesis and dust attenuation studies (Viaene et al. 2017b, 2018, 2019). The prospect of polarimetry at the DOT (Joshi et al. 2018) is also interesting for interstellar dust research (Peest et al. 2017).

The BINA collaboration can obviously be broadened to other telescopes at the disposal of the Belgian and Indian astronomical community. UGent astronomers have already used the GMRT radio interferometer for 21 cm Hi observations (Gentile et al. 2010; Allaert et al. 2015; Michałowski et al. 2016), and for radio continuum observations of radio galaxies (Nandi et al. 2014, 2017). A very interesting facility for interstellar dust studies is the Astrosat space mission (Singh et al. 2014); indeed, absorption and scattering at UV wavelengths is a useful way to characterise star formation and to discriminate between different dust models (Wijesinghe et al. 2011; Baes \& Viaene et al. 2016; Decleir et al. 2019). This possibility will be actively explored in the near future.

\section{Acknowledgements}

The authors thank the organisers of the 2nd BINA workshop for the invitation to present the UGent astronomy department. We acknowledge all colleagues at UGent for their magnificent work, which enabled us to write this overview.

\section{References}

Allaert F., Gentile G., Baes M. et al. 2015, A\&A, 582, A18

Allaert F., Gentile G., Baes M. 2017, A\&A, 605, A55

Amvrosiadis A., Eales S. A., Negrello M. et al. 2018, MNRAS, 475, 4939

Auld R., Minchin R. F., Davies J. I. et al. 2006, MNRAS, 371, 1617

Baes M., Camps P. 2015, Astronomy and Computing, 12, 33

Baes M., Dejonghe H. 2000, MNRAS, 313, 153

Baes M., Dejonghe H. 2001a, MNRAS, 326, 722

Baes M., Dejonghe H. 2001b, MNRAS, 326, 733

Baes M., Dejonghe H. 2001c, ApJL, 563, L19

Baes M., Dejonghe H. 2002a, MNRAS, 335, 441

Baes M., Dejonghe H. 2002b, A\&A, 393, 485

Baes M., Van Hese E. 2007, A\&A, 471, 419

Baes M., Van Hese E. 2011, A\&A, 534, A69

Baes M., Viaene S. 2016, A\&A, 587, A86

Baes M., Clemens M., Xilouris E. M. et al. 2010b, A\&A, 518, L53

Baes M., Davies J. I., Dejonghe H. et al. 2003, MNRAS, 343, 1081

Baes M., Dejonghe H., De Rijcke S. 2000, MNRAS, 318, 798

Baes M., Fritz J., Gadotti D. A. et al. 2010a, A\&A, 518, L39

Baes M., Allaert F., Sarzi M. et al. 2014, MNRAS, 444L, 90

Baes M., Gordon K. D., Lunttila T. et al. 2016, A\&A, 590, A55

Baes M., Stamatellos D., Davies J. I. et al. 2005, New Astronomy, 10, 523

Baes M., Trčka A., Camps P. et al. 2019, MNRAS, 484, 4069

Baes M., Verstappen J., De Looze I. et al. 2011, ApJS, 196, 22

Bakx T. J. L. C., Eales S. A., Negrello M. et al. 2018, MNRAS, 473, 1751

Bendo G. J., Wilson C. D., Pohlen M. et al. 2010, A\&A, 518, L65

Bendo G. J., Boselli A., Dariush A. et al. 2012, MNRAS, 419, 1833 
Bezanson R., van der Wel A., Pacifici C. et al. 2018a, ApJ, 858, 60

Bezanson R., van der Wel A., Straatman C. et al. 2018b, ApJL, 868, L36

Bianchi S., De Vis P., Viaene S. et al. 2018, A\&A, 620, A112

Bianchi S., Antonucci R., Capetti A. et al. 2019, MNRAS, 488L, 1

Boselli A., Eales S., Cortese L. et al. 2010, PASP, 122, 261

Boselli A., Ciesla L., Cortese L. et al. 2012, A\&A, 540, A54

Bussmann R. S., Gurwell M. A., Fu H. et al. 2012, ApJ, 756, 134

Buyle P., van Hese E., de Rijcke S., Dejonghe H. 2007, MNRAS, 375, 1157

Calanog J. A., Fu H., Cooray A. et al. 2014, ApJ, 797, 138

Camps P., Baes M. 2015, Astronomy and Computing, 9, 20

Camps P., Baes M., Saftly W. 2013, A\&A, 560, A35

Camps P., Misselt K., Bianchi S. et al. 2015, A\&A, 580, A87

Camps P., Trayford J. W., Baes M. et al. 2016, MNRAS, 462, 1057

Camps P., Trčka A., Trayford J. et al. 2018, ApJS, 234, 20

Cappellari M., Bacon R., Bureau M. et al. 2006, MNRAS, 366, 1126

Casasola V., Cassarà L. P., Bianchi S. et al. 2017, A\&A, 605, A18

Chauke P., van der Wel A., Pacifici C. et al. 2018, ApJ, 861, 13

Chung H., Ramaprakash A. N., Omar A. et al. 2014, Proc. SPIE, 9147, 91470V

Clark C. J. R., Verstocken S., Bianchi S. et al. 2018, A\&A, 609, A37

Cortese L., Minchin R. F., Auld R. R. et al. 2008, MNRAS, 383, 1519

Cortese L., Boissier S., Boselli A. et al. 2012, A\&A, 544A, 101

Davies J. I., Baes M., Bendo G. J. et al. 2010, A\&A, 518, L48

Davies J. I., Bianchi S., Cortese L. et al. 2012, MNRAS, 419, 3505

Davies J. I., Baes M., Bianchi S. et al. 2017, PASP, 129, 044102

de Blok W. J. G., Józsa G. I. G., Patterson M. et al. 2014, A\&A, 566, A80

De Bruyne V., Dejonghe H., Pizzella A., Bernardi M., Zeilinger W. W. 2001, ApJ, 546, 903

De Bruyne V., De Rijcke S., Dejonghe H., Zeilinger W. W. 2004, MNRAS, 349, 440

Decleir M., De Looze I., Boquien M. et al. 2019, MNRAS, in press (arXiv:1903.06715)

De Geyter G., Baes M., De Looze I. et al. 2015, MNRAS, 451, 1728

Dejonghe H. 1987, MNRAS, 224, 13

Dejonghe H. 1989, ApJ, 343, 113

Dejonghe H., de Bruyne V., Vauterin P., Zeilinger W. W. 1996, A\&A, 306, 363

De Looze I., Baes M., Bendo G. J. et al. 2012a, MNRAS, 427, 2797

De Looze I., Baes M., Fritz J., Verstappen J. 2012b, MNRAS, 419, 895

De Looze I., Baes M., Parkin T. J. et al. 2012c, MNRAS, 423, 2359

De Looze I., Fritz J., Baes M., et al. 2014, A\&A, 571, A69

De Rijcke S., Voulis I. 2016, MNRAS, 456, 2024

De Rijcke S., Dejonghe H., Zeilinger W. W., Hau G. K. T. 2001, ApJL, 559, L21

De Rijcke S., Michielsen D., Dejonghe H. et al. 2005, A\&A, 438, 491

De Rijcke S., Fouvry J.-B., Pichon C. 2019, MNRAS, 485, 150

Deschamps R., Braun K., Jorissen A. et al. 2015, A\&A, 577, A55

De Vis P., Dunne L., Maddox S. et al. 2017, MNRAS, 464, 4680

De Vis P., Jones A., Viaene S., et al. 2019, A\&A, 623, A5

Dunne L., Gomez H. L., da Cunha E. et al. 2011, MNRAS, 417, 1510

Dye S., Furlanetto C., Dunne L. et al. 2018, MNRAS, 476, 4383

Eales S., Dunne L., Clements D. et al. 2010, PASP, 122, 499

Emsellem E., Dejonghe H., Bacon R. 1999, MNRAS, 303, 495

Fernández-Ontiveros J. A., Armus L., Baes M. et al. 2017, PASA, 34, e053

Ford G. P., Gear W. K., Smith M. W. L. et al. 2013, ApJ, 769, 55

Fritz J., Gentile G., Smith M. W. L. et al. 2012, A\&A, 546A, 34

Gentile G., Baes M., Famaey B., van Acoleyen K. 2010, MNRAS, 406, 2493

Gentile G., Józsa G. I. G., Serra P. et al. 2013, A\&A, 554, A125

Gentile G., Tydtgat C., Baes M. et al. 2015, A\&A, 576, A57

Gordon K. D., Baes M., Bianchi S. et al. 2017, A\&A, 603, A114

Greenslade J., Clements D. L., Cheng T. et al. 2018, MNRAS, 476, 3336

Gruppioni C., Ciesla L., Hatziminaoglou E. et al. 2017, PASA, 34, e055

Heald G., Józsa G., Serra P. et al. 2011, A\&A, 526, A118

Hendrix T., Keppens R., Camps P. 2015, A\&A, 575, A110 
Hendrix T., Keppens R., van Marle A. J. et al. 2016, MNRAS, 460, 3975

Herranz D., González-Nuevo J., Clements D. L et al. 2013, A\&A, 549A, 31

Hughes T. M., Baes M., Fritz J. et al. 2014, A\&A, 565, A4

Hughes T. M., Baes M., Schirm M. R. P. et al. 2016, A\&A, 587, A45

Hughes T. M., Foyle K., Schirm M. R. P. et al. 2015, A\&A, 575, A17

Indebetouw R., Matsuura M., Dwek E. et al. 2014, ApJ, 782, 2

Joshi S., De Cat P. 2019, BSRSL, 88, 19 (this volume)

Joshi U. C., Ganesh S., Baliyan K. S. 2018, BSRSL, 87, 117

Koleva M., de Rijcke S., Prugniel P., Zeilinger W. W., Michielsen D. 2009, MNRAS, 396, 2133

Kumar B., Omar A., Maheswar G. et al. 2018, BSRSL, 87, 29

Lee D., Baes M., Seon K.-I. et al. 2016, MNRAS, 463, 2912

Liesenborgs J., De Rijcke S. 2012, MNRAS, 425, 1772

Liesenborgs J., De Rijcke S., Dejonghe H. 2006, MNRAS, 367, 1209

Liesenborgs J., de Rijcke S., Dejonghe H., Bekaert P. 2007, MNRAS, 380, 1729

Long R. J., Mao S. 2012, MNRAS, 421, 2580

Madden S. C., Rémy-Ruyer A., Galametz M. et al. 2013, PASP, 125, 600

Mathieu A., Dejonghe H., Hui X. 1996, A\&A, 309, 30

Michałowski M. J., Castro Cerón J. M., Wardlow J. L. et al. 2016, A\&A, 595, A72

Mosenkov A. V., Allaert F., Baes M. et al. 2016, A\&A, 592, A71

Mosenkov A. V., Allaert F., Baes M. et al. 2018, A\&A, 616, A120

Nandi S., Jamrozy M., Roy R. et al. 2017, MNRAS, 467, L56

Nandi S., Roy R., Saikia D. J. et al. 2014, ApJ, 789, 16

Negrello M., Amber S., Amvrosiadis A. et al. 2017, MNRAS, 465, 3558

Negrello M., Hopwood R., De Zotti G. et al. 2010, Science, 330, 800

Nersesian A., Xilouris E. M., Bianchi S. et al. 2019, A\&A, 624, A80

Peest C., Camps P., Stalevski M., Baes M., Siebenmorgen R. 2017, A\&A, 601, A92

Retana-Montenegro E., Frutos-Alfaro F., Baes M. 2012b, A\&A, 546, A32

Retana-Montenegro E., Van Hese E., Gentile G., Baes M., Frutos-Alfaro F. 2012a, A\&A, 540, A70

Saftly W., Baes M., Camps P. 2014, A\&A, 561, A77

Saftly W., Baes M., De Geyter G. et al. 2015, A\&A, 576, A31

Saftly W., Camps P., Baes M. et al. 2013, A\&A, 554, A10

Saglia R. P., Bertin G., Bertola F. et al. 1993, ApJ, 403, 567

Saintonge A., Wilson C. D., Xiao T. et al. 2018, MNRAS, 481, 3497

Schaye J., Crain R. A., Bower R. G. et al. 2015, MNRAS, 446, 521

Schroyen J., De Rijcke S., Koleva M., Cloet-Osselaer A., Vandenbroucke B. 2013, MNRAS, 434, 888

Schroyen J., De Rijcke S., Valcke S., Cloet-Osselaer A., Dejonghe H. 2011, MNRAS, 416, 601

Singh K. P., Tandon S. N., Agrawal P. C. et al. 2014, Proc. SPIE, 9144, 91441 S

Smith M. W. L., Eales S. A., Gomez H. L. et al. 2012, ApJ, 756, 40

Spilker J., Bezanson R., Barišić I. et al. 2018, ApJ, 860, 103

Spinoglio L., Alonso-Herrero A., Armus L. et al. 2017, PASA, 34, e057

Stalevski M., Fritz J., Baes M., Nakos T., Popović L. Č. 2012, MNRAS, 420, 2756

Stalevski M., Ricci C., Ueda Y. et al. 2016, MNRAS, 458, 2288

Stalevski M., Tristram K. R. W., Asmus D. 2019, MNRAS, 484, 3334

Statler T. S., Dejonghe H., Smecker-Hane T. 1999, AJ, 117, 126

Steinacker J., Baes M., Gordon K. D. 2013, ARA\&A, 51, 63

Straatman C. M. S., van der Wel A., Bezanson R. et al. 2018, ApJS, 239, 27

Timmons N., Cooray A., Riechers D. A. et al. 2016, ApJ, 829, 21

Trayford J. W., Camps P., Theuns T. et al. 2017, MNRAS, 470, 771

Valcke S., de Rijcke S., Dejonghe H. 2008, MNRAS, 389, 1111

van der Tak F. F. S., Madden S. C., Roelfsema, P. et al. 2018, PASA, 35, e002

van der Wel A., Noeske K., Bezanson R. et al. 2016, ApJS, 223, 29

Van Hese E., Baes M., Dejonghe H. 2009, ApJ, 690, 1280

Verbeke R., Papastergis E., Ponomareva A. A., Rathi S., De Rijcke S. 2017, A\&A, 607, A13

Verbeke R., Vandenbroucke B., De Rijcke S. 2015, ApJ, 815, 85

Verstappen J., Fritz J., Baes M. et al. 2013, A\&A, 556, A54

Verstocken S., Van De Putte D., Camps P., Baes M. 2017, Astronomy and Computing, 20, 16

Verstocken S., Viaene S., Nersesian A. et al. 2019, A\&A, submitted

Viaene S., Baes M., Bendo G. et al. 2016, A\&A, 586, A13 
Viaene S., Baes M., Tamm A. et al. 2017a, A\&A, 599, A64

Viaene S., Fritz J., Baes M. et al. 2014, A\&A, 567, A71

Viaene S., Sarzi M., Baes M., Fritz J., Puerari I. 2017b, MNRAS, 472, 1286

Viaene S., Sarzi M., Baes M., Puerari I. 2018, MNRAS, 474, L47

Viaene S., Sarzi M., Zabel N. et al. 2019, A\&A, 622, A89

Wijesinghe D. B., da Cunha E., Hopkins A. M. et al. 2011, MNRAS, 415, 1002

Wu P.-F., van der Wel A., Bezanson R. et al. 2018b, ApJ, 868, 37

Wu P.-F., van der Wel A., Gallazzi A. et al. 2018a, ApJ, 855, 85

Zhu L., Long R. J., Mao S. et al. 2014, ApJ, 792, 59 